\title{
Component structure of the vacant set induced by a random walk on a random graph.
}

\author{
Colin Cooper* $\quad$ Alan Frieze ${ }^{\dagger}$
}

June 20, 2011

\begin{abstract}
We consider random walks on several classes of graphs and explore the likely structure of the vacant set, i.e. the set of unvisited vertices. Let $\Gamma(t)$ be the subgraph induced by the vacant set of the walk at step $t$. We show that for random graphs $G_{n, p}$ (above the connectivity threshold) and for random regular graphs $G_{r}, r \geq 3$, the graph $\Gamma(t)$ undergoes a phase transition in the sense of the well-known Erdös-Renyi phase transition. Thus for $t \leq(1-\epsilon) t^{*}$, there is a unique giant component, plus components of size $O(\log n)$, and for $t \geq(1+\epsilon) t^{*}$ all components are of size $O(\log n)$. For $G_{n, p}$ and $G_{r}$ we give the value of $t^{*}$, and the size of $\Gamma(t)$. For $G_{r}$, we also give the degree sequence of $\Gamma(t)$, the size of the giant component (if any) of $\Gamma(t)$ and the number of tree components of $\Gamma(t)$ of a given size $k=O(\log n)$. We also show that for random digraphs $D_{n, p}$ above the strong connectivity threshold, there is a similar directed phase transition. Thus for $t \leq(1-\epsilon) t^{*}$, there is a unique strongly connected giant component, plus strongly connected components of size $O(\log n)$, and for $t \geq(1+\epsilon) t^{*}$ all strongly connected components are of size $O(\log n)$.
\end{abstract}

\section{Introduction}

The problem we consider can be described as follows. We have a finite graph $G=(V, E)$, and a simple random walk $\mathcal{W}_{u}$ on $G$, starting at $u \in V$. What is the likely component structure induced by the unvisited vertices of $G$ at step $t$ of the walk?

*Department of Informatics, King's College, University of London, London WC2R 2LS, UK

$\dagger$ Department of Mathematical Sciences, Carnegie Mellon University, Pittsburgh PA 15213, USA. Supported in part by NSF grant DMS0753472. 
Initially all vertices $V$ of $G$ are unvisited or vacant. We regard unvisited vertices as colored red. When $\mathcal{W}_{u}$ visits a vertex, the vertex is re-colored blue. Let $\mathcal{W}_{u}(t)$ denote the position of $\mathcal{W}_{u}$ at step $t$. Let $\mathcal{B}(t)=\left\{\mathcal{W}_{u}(0), \mathcal{W}_{u}(1), \ldots, \mathcal{W}_{u}(t)\right\}$ be the set of blue vertices at the end of step $t$, and $\mathcal{R}_{u}(t)=V \backslash \mathcal{B}_{u}(t)$. Let $\Gamma(t)$ be the subgraph of $G$ induced by $\mathcal{R}(t)$. Initially $\Gamma(0)$ is connected, unless $u$ is a cut-vertex. As the walk continues, $\Gamma(t)$ will shrink to the empty graph once every vertex has been visited. We wish to determine, as far as possible, the likely evolution of the component structure as $t$ increases. In this paper we will consider three models of random graphs, with vertex set $V=[n]$ where $[n]=\{1,2, \ldots, n\}$. These are the random graph $G_{n, p}$ in which each edge of $K_{n}$ is included independently with probability $p$, the random digraph $D_{n, p}$ in which each edge of $K_{n}$ is included independently with probability $p$ in each direction, and the random graph $G_{r}, r \geq 3$, sampled uniformly at random from the set of all simple $r$-regular graphs.

Because we consider random walks on random graphs, there are two sources of error in our proofs; (i) that we sample a graph $G$ which does not have the properties we need, or (ii) that the random walk $\mathcal{W}$ does not behave in the way we require. The set of graphs $\mathcal{G}^{\prime}$ with properties we require have measure $(1-o(1))$ of the graph space $\mathcal{G}$. Some of our proofs are for walks $\mathcal{W}$ on a fixed graph $G$ from the subset $\mathcal{G}^{\prime}$. In this case, walks $\mathcal{W}^{\prime}$ on $G$ with the properties we require have measure $(1-o(1))$ of $\mathcal{W}$. In other proofs, we use the method of deferred decisions, and reveal only the parts of the graph traversed by the walk. In that way, the vacant set $\mathcal{R}(t)$ of the walk induces a random graph, whose properties we can analyze.

Apart from $O(\cdot), o(\cdot), \Omega(\cdot)$ as a function of $n \rightarrow \infty$, where $n=|V|$, we use the following: We say $A_{n} \ll B_{n}$ or $B_{n} \gg A_{n}$ if $A_{n} / B_{n} \rightarrow 0$ as $n \rightarrow \infty$, and $A_{n} \sim B_{n}$ if $\lim _{n \rightarrow \infty} A_{n} / B_{n}=1$. The notation $\omega(n)$ describes a function tending to infinity as $n \rightarrow \infty$. We use the expression with high probability, whp, to mean, with probability $1-o\left(\log ^{-K} n\right)$ for any positive constant $K$. The variable $n$ is the size of the vertex set of the graph, and we measure both walk and graph probabilities in terms of this. Usually, it will be clear that we are discussing the graph, or the walk, but if we wish to stress this point we write $\mathbf{w h p}_{G}$ or $\mathbf{w h} \mathbf{p}_{\mathcal{W}}$. In the case where we use deferred decisions, if $|\mathcal{R}(t)|=N$, the whp statements are asymptotic in $N$, and we assume $N(n) \rightarrow \infty$. The statement of theorems in this section uses the annealed probability measure (graph and walk), i.e. whp relative to both graph sampling and walks.

We recall the typical evolution of the random graph $G_{n, p}$ as $p$ increases from 0 to 1 . Initially it consists of isolated vertices. As we increase $p$ or equivalently add random edges, we find that when $p=c / n$, the maximum component size is logarithmic for $c<1$, followed by a phase transition around the critical value $c=1$. When $c>1$ the maximum component size is linear in $n$, and all other components have logarithmic size. Our aim in this paper is to show that whp $\Gamma(t)$ undergoes a reversal of this. Thus $\Gamma(0)$ is connected and $\Gamma(t)$ starts to break up as $t$ increases. There is a critical value $t^{*}$ such that if $t<t^{*}$ by a sufficient amount then $\Gamma(t)$ consists of a unique giant component plus components of size $O(\log n)$. Once we have passed through the critical value, i.e. $t>t^{*}$ by a sufficient amount, then all components are of size $O(\log n)$. As $t$ increases, the maximum component size shrinks to zero. We make the 
following definitions. A graph with vertex set $V_{1}$ is sub-critical if its maximum component size is $O(\log n)$, and super-critical if is has a unique component $C_{1}(t)$ of size $\Omega\left(\left|V_{1}(t)\right|\right)$, where $\left|V_{1}(t)\right| \gg \log n$, and all other components are of size $O(\log n)$.

In the case of random digraphs $D_{n, p}$ the evolution is more complex. Let $q=1-(1-p)^{2}$. There is a phase transition around $q=1 / n$ (i.e. $p \sim 1 / 2 n$ ) for the emergence of a giant component in the underlying graph $G_{n, q}$ of $D_{n, p}$, and, around $p=1 / n$ (i.e. $q \sim 2 / n$ ) for the emergence of a giant strongly connected component in $D_{n, p}$. We make the following definitions. A digraph with vertex set $V_{1}$ is directed-sub-critical if its maximum strongly connected component size is $O(\log n)$, and directed-super-critical if is has a unique strongly connected component $C_{1}(t)$ of size $\Omega\left(\left|V_{1}(t)\right|\right)$, where $\left|V_{1}(t)\right| \gg \log n$, and all other strongly connected components are of size $O(\log n)$.

Component structure of vacant set of random graphs $G_{n, p}$ and $D_{n, p}$. We first consider $G_{n, p}$. We assume that

$$
n p=c \log n \text { where }(c-1) \log n \rightarrow \infty \text { with } n, \quad \text { and } c=n^{1 / \omega} .
$$

where $\omega=\omega(n)$ can be any function tending to infinity with $n$.

Let

$$
t_{\theta}=n(\log \log n+(1+\theta) \log c) .
$$

Theorem 1. Let $\epsilon>0$ be a small constant. Then whp in $G_{n, p}$ we have (i) $\Gamma(t)$ is supercritical for $t \leq t_{-\epsilon}$, (ii) $\Gamma(t)$ is sub-critical for $t \geq t_{\epsilon}$.

In Section 5, we prove that for $t=t_{\theta}$, whp the size of $\mathcal{R}(t)$ is $N\left(t_{\theta}\right)=(1+o(1)) n /\left(c^{1+\theta} \log n\right)$, and that $\Gamma(t)$ behaves as the random graph $G_{N\left(t_{\theta}\right), p}$. The threshold for the giant component in $G_{N, p}$ is at $N p=1$. For $\theta<0$, let $\theta=-\epsilon$. Then $N\left(t_{-\epsilon}\right) p=c^{\epsilon}>1$, and there is a giant component $C_{1}\left(t_{-\epsilon}\right)$ of size $\Omega\left(N\left(t_{-\epsilon}\right)\right)=\Omega(n /(c \log n))$. The bound $c=n^{1 / \omega}$, ignores larger values of $p$. On the other hand there is not going to be a phase transition as $p \rightarrow 1 / \log n$.

We next consider $D_{n, p}$. Let $q=1-(1-p)^{2}$. Let $\vec{\Gamma}(t)$ be the directed graph induced by the vacant set of $D_{n, p}$ and let $\Gamma(t)$ be the undirected graph induced by the vacant set of the underlying graph $G_{n, q}$ of $D_{n, p}$. Theorem 1 holds unaltered for $\Gamma(t)$, with $n q=c \log n$. For strongly connected components of $\vec{\Gamma}(t)$, we have the following theorem.

Theorem 2. Let $\epsilon>0$ be a small constant. Let $n p=c \log n$. Then whp in $D_{n, p}$ we have (i) $\vec{\Gamma}(t)$ is directed-super-critical for $t \leq t_{-\epsilon}$, (ii) $\vec{\Gamma}(t)$ is directed-sub-critical for $t \geq t_{\epsilon}$.

\section{Component structure of vacant set of random regular graphs.}

We next consider $G_{r}$ for $r \geq 3$, constant. Let

$$
t^{*}=\frac{r(r-1) \log (r-1)}{(r-2)^{2}} n .
$$


Let

$$
N_{t}=n e^{-\frac{(r-2) t}{(r-1) n}}=n e^{-\frac{t}{\rho n}},
$$

where $\rho=(r-1) /(r-2)$, and let

$$
p_{t}=e^{-\frac{(r-2) t}{\rho r n}} .
$$

Theorem 3. Let $\epsilon>0$ be a small constant. Then whp we have

(i) $\Gamma(t)$ is super-critical for $t \leq(1-\epsilon) t^{*}$, and $\left|C_{1}(t)\right|=\Omega(n)$. Let $p=p_{t}$, then

$$
\left|C_{1}(t)\right| \sim \theta N_{t}
$$

where,

$$
\theta=1-\left(1-p+p \phi^{1 / 2}\right)^{r}
$$

and where $\phi$ is the largest positive solution in $(0,1)$ to

$$
\phi=\left(1-p+p \phi^{1 / 2}\right)^{2(r-1)} .
$$

(ii) $\Gamma(t)$ is sub-critical for $t \geq(1+\epsilon) t^{*}$,

(iii) At some time $t \sim t^{*}$ the maximum component size in $\Gamma(t)$ is $n^{2 / 3+o(1)}$.

When $r=3$, equations (4), (5) give $\phi=((1-p) / p)^{4}$ and $\theta=1-\left(e^{t /(6 n)}-1\right)^{3}$.

We can also say something about $|\mathcal{R}(t)|$ and the degree sequence of $\Gamma(t)$.

Let $\tau_{0}=0$ and for $k \geq 1$ let

$$
\tau_{k}=n^{1-1 / k}, \quad \text { and } \quad t_{k}=\frac{\rho r n \log n}{k(r-2)+r} .
$$

Theorem 4. Let $\epsilon, \delta$ be small positive constants. Suppose that $\log \log n \ll \omega=\omega(n) \ll \log n$.

(a) whp simultaneously for all $t \leq(1-\epsilon) t_{0},|\mathcal{R}(t)|=(1+o(1)) N_{t}$. This result also holds whp for any fixed $(1-\epsilon) t_{0} \leq t \leq t_{0}-\omega n$.

(b) Let $D_{s}(t)$ be the number of vertices of degree $s$ in $\Gamma(t)$. Then $\mathbf{w h p}$, simultaneously for all $0 \leq s \leq r$ and for all $\left(\tau_{r-s}\right)^{1+\delta} \leq t \leq(1-\epsilon) t_{s}$,

$$
D_{s}(t)=(1+o(1)) N_{t}\left(\begin{array}{l}
r \\
s
\end{array}\right) p_{t}^{s}\left(1-p_{t}\right)^{r-s} .
$$

This result also holds whp for any fixed $(1-\epsilon) t_{s} \leq t \leq t_{s}-\omega n$. 
We make the following remarks.

- It was proved in [7] that the cover time of $G_{r}$ is $C\left(G_{r}\right) \sim((r-1) /(r-2)) n \log n=t_{0}$.

- The whp concentration of e.g. $|\mathcal{R}(t)|$ in Theorem 4 holds simultaneously for all $t \leq$ $(1-\epsilon) t_{0}$. The value of $\epsilon$ can be made arbitrarily small. We have not attempted to optimize these results. It is also true for any given $t \leq t_{0}-\omega n$, but not proven to hold for every $t$.

- The range $t_{s}-\omega n \leq t \leq t_{s}+\omega n$ contains the times when the number of vertices of degree $s$ is constant in expectation and unlikely to be concentrated around its mean.

We can give some more information about the number of small components in $\Gamma(t)$. Again there is a gap containing the times when the expected number of such components is constant.

Theorem 5. Let $\epsilon$ be a small positive constant. Let

$$
\eta(k, t)=n e^{-t /(\rho n)} \frac{r}{k((r-2) k+2)}\left(\begin{array}{c}
(r-1) k \\
k-1
\end{array}\right) p_{t}^{k-1}\left(1-p_{t}\right)^{k(r-2)+2} .
$$

(a) Let $1 \leq k \leq \epsilon \log n$ and $\epsilon n \leq t \leq(1-\epsilon) t_{k-1}$. Let $N(k, t)$ denote the number of tree components of $\Gamma(t)$ with $k$ vertices. Then $\mathbf{w h p}$ for any given $t, N(k, t)=(1+o(1)) \eta(k, t)$.

(b) For $k$ constant, whp, simultaneously for all $t$ in the range $\epsilon n \leq t \leq(1-\epsilon) t_{k-1}$, the number of trees with $k$ vertices is concentrated around the value $\eta(k, t)$. This result also holds whp for any given $t$ in the range $\left(\tau_{k(r-2)+2}\right)^{1+\delta} \leq t \leq \epsilon n$, where $\delta$ is a small positive constant.

Again we make some remarks.

- Most small components are trees, as e.g. whp $G_{r}$ induces at most $O(\log n)$ cycles of constant size.

- Theorem 5 holds simultaneously for $k$ constant and any $t$. We do not claim that Theorem 5 holds simultaneously for all $k=O(\log n)$ and $t$. Our proof only show this to be true for most values of $t$.

- The intervals containing trees of size $k$ constant are nested. Thus, whp isolated red vertices are the first trees to appear, (at around time $n^{1-1 / r}$ ), and the last to disappear, at the cover time (around $t_{0}=\rho n \log n$ ). 
Finally, we state without detailed proof the results on the vacant set arising from the use of $k$ simultaneous random walks, $k \geq 1$ constant. The structure of $\Gamma_{k}(t)$ is equivalent to $\Gamma\left(t^{\prime}\right)$ where $t^{\prime}=(1+o(1)) k t$. In particular $t_{k}^{*}=t^{*} / k$. The reasons for this assertion are based on Lemma 9 and Corollary 10. The probability that none of $k$ independent random walks visit a vertex $v$ during steps $T, \ldots, t$, is the $k$-th power of the probability that a single random walk does not visit $v$ during steps $T, \ldots, t$.

\subsection{Previous work}

The only previous works on this subject that we are aware of are Benjamini and Sznitman [1], Windisch [21] and Černy, Teixeira and Windisch [5]. Papers [1], [21] deal with the component structure of the vacant set of a random walk on a $d$-dimensional torus. Paper [5] deals with random walks on $G_{r}$. It shows that whp $\Gamma(t)$ is sub-critical for $t \geq(1+\epsilon) t^{*}$ and that there is a unique linear size component for $t \leq(1-\epsilon) t^{*}$. They conjecture that $\Gamma(t)$ is super-critical for $t \leq(1-\epsilon) t^{*}$ and we prove this conjecture.

Subsequent to our posting a preliminary version on the ArXiV, Cerny and Teixeira [6] have used the methods of this paper to give a sharper analysis of $\Gamma(t)$ in the critical window.

\section{Uniformity}

The method we use to study the vacant set, uses the random walk to generate the graph $G$ in question. The main idea is to realize that as $G$ is a random graph, the graph $\Gamma(t)$ of the vacant set has a simple description.

We first consider $G_{n, p}$. To prove Theorem 1 we only need high probability estimates of $|\mathcal{R}(t)|$.

\section{Lemma 6.}

Consider a random walk on $G_{n, p}$. Conditional on $N=|\mathcal{R}(t)|, \Gamma(t)$ is distributed as $G_{N, p}$.

Proof This follows easily from the principle of deferred decisions. We do not have to decide the existence or absence of edges between vertices in $\mathcal{R}(t)$ until one of them is exposed.

We next consider $G_{r}$. We give two structural lemmas.

\section{Lemma 7.}

Consider a random walk on $G_{r}$. Let $\Gamma(t)$ have vertex set $\mathcal{R}(t)$ and degree sequence $\mathbf{d}=$ $\left(d_{\Gamma(t)}(v), v \in \mathcal{R}(t)\right)$. Conditional on $N=|\mathcal{R}(t)|$ and degree sequence $\mathbf{d}, \Gamma(t)$ is distributed as $G_{N, \mathbf{d}}$, the random graph with vertex set $[N]$ and degree sequence $\mathbf{d}$. 
Proof Intuitively, if we condition on $\mathcal{R}(t)$ and the history of the process, and if $G_{1}, G_{2}$ are graphs with vertex set $\mathcal{R}(t)$ and if they have the same degree sequence, then substituting $G_{2}$ for $G_{1}$ will not conflict with the history. Every extension of $G_{1}$ is an extension of $G_{2}$ and vice-versa. We now give a formal explanation of this.

The history of the process can be represented by a sequence $X=\left(X_{0}, \ldots, X_{t-1}\right)$ where $X \in[r]^{t}$. This sequence is to be interpreted as follows. We assume the neighbours $u_{1}, \ldots, u_{r}$ of each vertex $v$ are given in some fixed order, e.g. increasing label order, (recall that $V=[n]$ ). When at the $j$-th vertex $v=\mathcal{W}_{u}(j-1)$, the walk moved to the $X_{j}$-th neighbour $u_{X_{j}}$ of $v$, in the given order. The probability space for the lemma is uniform over $\mathcal{G}_{r} \times[r]^{t}$, where $\mathcal{G}_{r}$ is the set of $r$-regular graphs on $[n]$. Given $\omega=(G, X)$, we let $\mathcal{R}_{\omega}, \mathcal{B}_{\omega}, E_{\omega}, \mathbf{d}_{\omega}$ denote the induced values of $\mathcal{R}(t), \mathcal{B}(t)$, the edges of $G$ that are incident with $\mathcal{B}(t)$ and the degree sequence of the graph $\Gamma_{\omega}$ induced by $\mathcal{R}(t)$. These quantities are all determined by $\omega$. For consistency with the statement of the Lemma, when $N=|\mathcal{R}(t)|$, let $d_{\omega}(i)$ be the degree of the $i$-th vertex of $\Gamma_{\omega}$ in numerical order. We can in this way associate $\Gamma_{\omega}$ with $G_{N, \mathbf{d}}$.

Fix $\mathcal{R}$ and a degree sequence $\mathbf{d}$. Let $\mathcal{G}_{\mathcal{R}, \mathbf{d}}$ be the set of graphs with vertex set $\mathcal{R}$ and degree sequence d. For a graph $H \in \mathcal{G}_{\mathcal{R}, \mathbf{d}}$ and $X \in[r]^{t}$ we let $\Psi_{X}(H)=\left\{\omega=(G, X): \Gamma_{\omega}=H\right\}$ and $\Omega_{X}(H)=\left\{G:(G, X) \in \Psi_{X}(H)\right\}$. Note that $\left|\Psi_{X}(H)\right|=\left|\Omega_{X}(H)\right|$. Now for any fixed $X_{0} \in[r]^{t}$,

$$
\operatorname{Pr}\left(\Gamma_{\omega}=H \mid X=X_{0}\right)=\frac{\operatorname{Pr}\left(\left(\Gamma_{\omega}=H\right) \wedge\left(X=X_{0}\right)\right)}{\operatorname{Pr}\left(X=X_{0}\right)}=\frac{\left|\Psi_{X_{0}}(H)\right| /\left(\left|\mathcal{G}_{r}\right| r^{t}\right)}{\operatorname{Pr}\left(X=X_{0}\right)}=\frac{\left|\Psi_{X_{0}}(H)\right|}{\left|\mathcal{G}_{r}\right|} .
$$

We argue next that for given $X$ and $H_{1}, H_{2} \in \mathcal{G}_{\mathcal{R}, \mathbf{d}}$ we have $\left|\Omega_{X}\left(H_{1}\right)\right|=\left|\Omega_{X}\left(H_{2}\right)\right|$. The lemma follows from this and (7).

For $G \in \Omega_{X}\left(H_{1}\right)$ let $\phi(G)=\phi_{H_{1}, H_{2}}(G)=\left(G \backslash E\left(H_{1}\right)\right) \cup E\left(H_{2}\right)$, i.e. we remove the edges of $H_{1}$ from $G$ and replace them by the edges of $H_{2}$. Note first that $\phi(G) \in \mathcal{G}_{r}$, and that if $\phi(G)=\phi\left(G^{\prime}\right)$ then $G=G^{\prime}$. We next show that if $G \in \Omega_{X}\left(H_{1}\right)$ then $\phi(G) \in \Omega_{X}\left(H_{2}\right)$, in which case, because $\phi$ is bijective, we have $\left|\Omega_{X}\left(H_{1}\right)\right|=\left|\Omega_{X}\left(H_{2}\right)\right|$.

Given $\omega_{1}=(G, X)$, let $\omega_{2}=(\phi(G), X)$. Since we have not changed $X$ or $G \backslash E\left(H_{1}\right)$, the walk $\mathcal{W}_{u}(s)$ described by $X$ made the same edge transitions at steps $0 \leq s \leq t-1$, on $G$ and $\phi(G)$. This means that $\mathcal{B}_{\omega_{2}}=\mathcal{B}_{\omega_{1}}$, and hence $\mathcal{R}_{\omega_{1}}=\mathcal{R}_{\omega_{2}}$. Thus $\Gamma_{\omega_{2}}=H_{2}$, so that $\phi(G) \in \Omega_{X}\left(H_{2}\right)$. The lemma follows.

Thus to prove Theorem 3 we only need high probability estimates of the degree sequence of $\Gamma(t)$. The proof of Theorem 5 can in principle be derived from this, although we do not have a simple way of doing it. Instead we rely on a further characterization of $\Gamma(t)$.

We use the configuration or pairing model of Bollobás [3] and Bender and Canfield [4]. We start with $n$ disjoint sets of points $W_{1}, W_{2}, \ldots, W_{n}$ each of size $r$. We let $W=\bigcup_{i=1}^{n} W_{i}$. A configuration $F$ is a partition of $W$ into $r n / 2$ pairs i.e. a pairing. $\Omega$ is the set of 
configurations. If $F \in \Omega$ defines an $r$-regular multi-graph $G_{F}=\left([n], E_{F}\right)$ where $E_{F}=$ $\left\{(i, j): \exists\{x, y\} \in F: x \in W_{i}, y \in W_{j}\right\}$ i.e. we contract $W_{i}$ to a vertex $i$ for $i \in[n]$.

It is known that: (i) Each simple graph arises the same number of times as $G_{F}$. i.e. if $G, G^{\prime}$ are simple, then $\left|\left\{F: G_{F}=G\right\}\right|=\left|\left\{F^{\prime}: G_{F}^{\prime}=G^{\prime}\right\}\right|$. (ii) Provided $r$ is constant, the probability $G_{F}$ is simple is bounded below by a constant. Thus if $F$ is chosen uniformly at random from $\Omega$ then any event that occurs whp for $F$, occurs whp for $G_{F}$, and hence whp for $G_{r}$.

Suppose now that we generate a random $F$ using a random walk on $[n]$. To do this, we begin with a starting vertex $i_{1}$, and at the start of $t$-th step we are at some vertex $i_{t}$, and have a partition $R_{t}, B_{t}$ of $W$ into Red and Blue points respectively. Initially, $R_{1}=W$ and $B_{1}=\emptyset$. In addition we have a collection $F_{t}$ of disjoint pairs from $W$ where $F_{1}=\emptyset$.

At step $t+1$ we choose a random edge incident with $i_{t}$. Recall that the neighbours of $i_{t}$ are in a fixed order indexed by $1, \ldots, r$. Obviously $i_{t} \in \mathcal{B}(t)$, as it is visited by the walk, but we treat the configuration points in $W_{i_{t}}$ as blue or red, depending on whether the corresponding edge is previously traversed (blue) or not (red). Let $x$ be chosen randomly from $W_{i_{t}}$. There are two cases.

If $x \in R_{t}$, then the edge is unvisited, so we choose $y$ randomly from $R_{t} \backslash\{x\}$. Suppose that $y \in W_{j}$. This is equivalent to moving from $i_{t} \in \mathcal{B}(t)$ to $i_{t+1}=j$. If $j \in \mathcal{B}(t)$ this is equivalent to moving between blue vertices on a previously unvisited edge. If $j \in \mathcal{R}(t)$, this is equivalent to moving to a previously unvisited vertex. We update as follows. $R_{t+1}=R_{t} \backslash\{x, y\}$ and $B_{t+1}=B_{t} \cup\{x, y\}$, and $F_{t+1}=F_{t} \cup\{\{x, y\}\}$.

If on the other hand, $x \in B_{t}$ then it has previously been paired with a $y \in W_{j} \cap B_{t}$ and we move from $i_{t}$ to $i_{t+1}=j$ without updating. We let $R_{t+1}=R_{t}, B_{t+1}=B_{t}$ and we let $F_{t+1}=F_{t}$.

After $t$ steps we will have constructed a random collection $F_{t}$ of at most $t$ disjoint pairs from $W$. $F_{t}$ consists of a pairing of $B_{t}$, and $R_{t}$ is unpaired. In principle we can extend $F_{t}$ to a random configuration $F$ by adding a random pairing of $R_{t}$ to it. The next lemma summarizes this discussion.

\section{Lemma 8.}

(a) $F_{t}$ plus a random pairing of $R_{t}$ is a uniform random member of $\Omega$.

(b) $i \in \mathcal{R}(t)$ iff $W_{i} \subseteq R_{t}$. 


\section{$3 \quad$ Vacancy probabilities}

As in our previous papers on random walks on random graphs, we make heavy use of Lemma 9 below. Let $P$ be the transition matrix of the walk and let $P_{u}^{(t)}(v)=\operatorname{Pr}\left(\mathcal{W}_{u}(t)=v\right)$ be the $t$-step transition probability. We assume the random walk $\mathcal{W}_{u}$ on $G$ is ergodic, and thus the random walk has stationary distribution $\pi$, where $\pi_{v}=d(v) /(2 m)$.

Suppose that the eigenvalues of $P$ are $1=\lambda_{1}>\lambda_{2} \geq \cdots \geq \lambda_{n}$. Let $\lambda_{\max }=\max \left\{\left|\lambda_{i}\right|: i \geq 2\right\}$. We can make $\lambda_{2}=\lambda_{\max }$, if necessary, by making the chain lazy i.e. by not moving with probability $1 / 2$ at each step. This has no significant effect on the analysis. Let $\Phi_{G}$ be the conductance of $G$ i.e.

$$
\Phi_{G}=\min _{S \subseteq V, \pi_{S} \leq 1 / 2} \frac{\sum_{x \in S} \pi_{x} P(x, \bar{S})}{\pi_{S}} .
$$

Then,

$$
\begin{aligned}
& 1-\Phi_{G} \leq \lambda_{2} \leq 1-\frac{\Phi_{G}^{2}}{2} \\
& \left|P_{u}^{(t)}(x)-\pi_{x}\right| \leq\left(\pi_{x} / \pi_{u}\right)^{1 / 2} \lambda_{\max }^{t}
\end{aligned}
$$

A proof of this can be found for example in Jerrum and Sinclair [16].

Mixing time of $G_{n, p}, G_{r}$. Let $T$ be such that, for $t \geq T$

$$
\max _{u, x \in V}\left|P_{u}^{(t)}(x)-\pi_{x}\right|=O\left(\frac{\min _{x \in V} \pi_{x}}{n^{3}}\right) .
$$

For $G=G_{n, p}$ and $n p=c \log n, c>1$, whp the conductance $\Phi(G)>0$ constant, and so

$$
T\left(G_{n, p}\right)=O(\log n) .
$$

For $G=G_{r}$, Friedman [13] has shown that whp $\lambda_{2} \leq(2 \sqrt{r-1}+\epsilon) / r \leq 29 / 30$, say. In which case we can whp take

$$
T\left(G_{r}\right) \leq 120 \log n
$$

If inequality (11) holds, we say the distribution of the walk is in near stationarity. Fix two vertices $u, v$. Let $h_{t}=\operatorname{Pr}\left(\mathcal{W}_{u}(t)=v\right)$ be the probability that the walk $\mathcal{W}_{u}$ visits $v$ at step $t$. Let

$$
H(z)=\sum_{t=T}^{\infty} h_{t} z^{t}
$$

generate $h_{t}$ for $t \geq T$. 
We next consider the returns to vertex $v$ made by a walk $\mathcal{W}_{v}$, starting at $v$. Let $r_{t}=$ $\operatorname{Pr}\left(\mathcal{W}_{v}(t)=v\right)$ be the probability that the walk returns to $v$ at step $t=0,1, \ldots$ In particular note that $r_{0}=1$, as the walk starts on $v$. Let

$$
R(z)=\sum_{t=0}^{\infty} r_{t} z^{t}
$$

generate $r_{t}$, and let

$$
R_{T}(z)=\sum_{j=0}^{T-1} r_{j} z^{j} .
$$

Thus, evaluating $R_{T}(z)$ at $z=1$, we have $R_{T}(1) \geq r_{0}=1$.

For $t \geq T$ let $f_{t}=f_{t}(u \rightarrow v)$ be the probability that the first visit made to $v$ by the walk $\mathcal{W}_{u}$ to $v$ in the period $[T, T+1, \ldots]$ occurs at step $t$. Let

$$
F(z)=\sum_{t=T}^{\infty} f_{t} z^{t}
$$

generate $f_{t}$. Then we have

$$
H(z)=F(z) R(z)
$$

The following lemma gives the probability that a walk, starting from near stationarity makes a first visit to vertex $v$ at a given step. For proofs of the lemma and its corollary, see [9]. The proof differs from the earlier version given in [7], in that we only consider first visits to a vertex $v$ after the mixing time $T$. We use the lemma to estimate $\mathbf{E}\left|\mathcal{R}_{T}(t)\right|$, the expected number of vertices unvisited after $T$, this differs from $\mathbf{E}|\mathcal{R}(t)|$ by at most $T$ vertices.

Lemma 9. Let $R_{v}=R_{T}(1)$, where $R_{T}(z)$ is from (15). For some sufficiently large constant $K$, let

$$
\lambda=\frac{1}{K T},
$$

where $T$ satisfies (11). Suppose that

(i) For some constant $\theta>0$, we have

$$
\min _{|z| \leq 1+\lambda}\left|R_{T}(z)\right| \geq \theta .
$$

(ii) $T \pi_{v}=o(1)$ and $T \pi_{v}=\Omega\left(n^{-2}\right)$.

There exists

$$
p_{v}=\frac{\pi_{v}}{R_{v}\left(1+O\left(T \pi_{v}\right)\right)},
$$


such that for all $t \geq T$,

$$
\begin{aligned}
f_{t}(u \rightarrow v) & =\left(1+O\left(T \pi_{v}\right)\right) \frac{p_{v}}{\left(1+p_{v}\right)^{t+1}}+O\left(T \pi_{v} e^{-\lambda t / 2}\right) . \\
& =\left(1+O\left(T \pi_{v}\right)\right) \frac{p_{v}}{\left(1+p_{v}\right)^{t}} \quad \text { for } t \geq \log ^{3} n .
\end{aligned}
$$

Corollary 10. Fort $\geq T$ let $\mathcal{A}_{v}(t)$ be the event that $\mathcal{W}_{u}$ does not visit $v$ at steps $T, T+1, \ldots, t$. Then, under the assumptions of Lemma 9,

$$
\begin{aligned}
\operatorname{Pr}_{\mathcal{W}}\left(\mathcal{A}_{v}(t)\right) & =\frac{\left(1+O\left(T \pi_{v}\right)\right)}{\left(1+p_{v}\right)^{t}}+O\left(T^{2} \pi_{v} e^{-\lambda t / 2}\right) \\
& =\frac{\left(1+O\left(T \pi_{v}\right)\right)}{\left(1+p_{v}\right)^{t}} \quad \text { for } t \geq \log ^{3} n .
\end{aligned}
$$

We use the notation $\operatorname{Pr}_{\mathcal{W}}$ when we want to emphasize that we are dealing with the probability space of walks on $G$.

Corollary 10 gives the probability of not visiting a single vertex in time $[T, t]$. We need to extend this result to certain small sets of vertices. In particular we need to consider sets consisting of $v$ and a subset of its neighbours $N(v)$. Let $S$ be such a subset.

Suppose now that $S$ is a subset of $V$ with $|S|=o(n)$. By contracting $S$ to single vertex $\gamma=\gamma(S)$, we form a graph $H=H(S)$ in which the set $S$ is replaced by $\gamma$ and the edges that were contained in $S$ are contracted to loops. The probability of no visit to $S$ in $G$ can be found (up to a multiplicative error of $1+O\left(1 / n^{3}\right)$ ) from the probability of a first visit to $\gamma$ in $H$. This is the content of Lemma 11 below.

We can estimate the mixing time of a random walk on $H$ as follows. Note that the conductance of $H$ is at least that of $G$. As some subsets of vertices of $V$ have been removed by the contraction of $S$, the set of values that we minimise over, to calculate the conductance of $H$, (see (8)), is a subset of the set of values that we minimise over for $G$. It follows that the conductance of $H$ is bounded below by the conductance of $G$. The conductance of $G$ is constant, (see discussion below (11)), and so using (9), (10), we see that the mixing time for $\mathcal{W}$ in $H$ is $O(\log n)$.

Lemma 11. [9] Let $\mathcal{W}_{u}$ be a random walk in $G$ starting at $u \notin S$, and let $\mathcal{X}_{u}$ be a random walk in $H$ starting at $u \neq \gamma$. Let $T$ be a mixing time satisfying (11) in both $G$ and $H$. Then

$$
\operatorname{Pr}\left(\mathcal{A}_{\gamma}(t) ; H\right)=\operatorname{Pr}\left(\wedge_{v \in S} \mathcal{A}_{v}(t) ; G\right)\left(1+O\left(\frac{1}{n^{3}}\right)\right),
$$

where the probabilities are those derived from the walk in the given graph. 


\section{Proof}

Note that $m=r n / 2=|E(G)|=|E(H)|$. Let $\mathcal{W}_{x}(j)$ (resp. $\left.X_{x}(j)\right)$ be the position of walk $\mathcal{W}_{x}\left(\operatorname{resp} . \mathcal{X}_{x}(j)\right)$ at step $j$. Let $\Gamma=G, H$ and let $P_{u}^{s}(x ; \Gamma)$ be the transition probability in $\Gamma$, for the walk to go from $u$ to $x$ in $s$ steps.

$$
\begin{aligned}
\operatorname{Pr}\left(\mathcal{A}_{\gamma}(t) ; H\right) & =\sum_{x \neq \gamma} P_{u}^{T}(x ; H) \operatorname{Pr}\left(X_{x}(s-T) \neq \gamma, T \leq s \leq t ; H\right) \\
& =\sum_{x \neq \gamma}\left(\frac{d(x)}{2 m}\left(1+O\left(n^{-3}\right)\right)\right) \operatorname{Pr}\left(X_{x}(s-T) \neq \gamma, T \leq s \leq t ; H\right) \\
& =\sum_{x \notin S}\left(P_{u}^{T}(x ; G)\left(1+O\left(n^{-3}\right)\right)\right) \operatorname{Pr}\left(\mathcal{W}_{x}(s-T) \notin S, T \leq s \leq t ; G\right) \\
& =\operatorname{Pr}\left(\wedge_{v \in S} \mathcal{A}_{v}(t) ; G\right)\left(1+O\left(1 / n^{3}\right)\right) .
\end{aligned}
$$

Equation (23) follows from (11). Equation (24) follows because there is a natural measure preserving map $\phi$ between walks in $G$ that start at $x \notin S$ and avoid $S$ and walks in $H$ that start at $x \neq \gamma$ and avoid $\gamma$.

\section{The evolution of $\Gamma(t)$ in $G_{r}$}

\subsection{Estimates of $R_{v}$}

Let

$$
\ell_{1}=\epsilon_{1} \log _{r} n,
$$

for some sufficiently small $\epsilon_{1}$. A cycle $C$ is small if $|C| \leq \ell_{1}$. A vertex is nice if it is at distance at least $\ell_{1}$ from any small cycle. Let $\mathcal{N}$ denote the nice vertices and $\overline{\mathcal{N}}$ denote the vertices that are not nice.

It is straightforward to prove by first moment calculations that:

Whp there are at most $n^{2 \epsilon_{1}}$ vertices that are not nice.

Whp there are no two small cycles within distance $\ell_{1}$ of each other.

The results we prove are all conditional on (26) and (27) holding. This can only inflate the probabilities of unlikely events by $1+o(1)$. This includes events defined in terms of the configuration model as claimed in Lemma 8. For example, if a calculation shows that an event $\mathcal{E}$ has probability at most $\epsilon$ in the configuration model, then it has probability $O(\epsilon)$ with respect to the corresponding subgraph of $G$ and then we only need to multiply this by $1+o(1)$ in order to estimate the probability conditional on (26) and (27). We will continue relying on this without further comment. 
A vertex $v$ is tree-like to depth $k$ if $N_{k}(v)$ induces a tree, rooted at $v$. Here $N_{k}(v)$ denotes the set of vertices at distance at most $k$ from $v, k \geq 1$. Thus a nice vertex is tree-like to depth $\ell_{1} / 2$.

\section{Lemma 12.}

(a) If $v$ is nice then

$$
R_{v}=(1+o(1)) \rho \text { where } \rho=\frac{r-1}{r-2},
$$

where the $o(1)$ term is $o\left(\log ^{-K} n\right)$ for any positive constant $K$.

(b) If $v$ is not nice then

$$
R_{v} \leq(1+o(1)) \frac{r}{r-2}
$$

Proof (a) Let $H$ denote the subgraph of $G$ induced by $N_{\ell_{1} / 2}(v)$. This is a tree and we can embed it into an infinite $r$-regular tree $\mathcal{T}$ rooted at $v$. Let $\mathcal{X}$ be the walk on $\mathcal{T}$, starting from $v$, and let $X_{t}$ be the distance of $\mathcal{X}$ from the root vertex at step $t$.

Let $D_{0}=0$, and let $D_{t}$ be the distance from $v$ of $\mathcal{W}$ in $G$ at step $t$. We note that we can couple $\mathcal{W}_{v}, \mathcal{X}$ so that $D_{t}=X_{t}$ up until the first time that $D_{t}>\ell_{1} / 2$.

The values of $X_{t}$ are as follows: $X_{0}=0, X_{1}=1$, and if $X_{t}=0$ then $X_{t+1}=1$. If $X_{t}>0$ then

$$
X_{t}= \begin{cases}X_{t-1}-1 & \text { with probability } q=\frac{1}{r} \\ X_{t-1}+1 & \text { with probability } p=\frac{r-1}{r} .\end{cases}
$$

We note the following result (see e.g. [12]), for a random walk on the line $=\{0, \ldots, a\}$ with absorbing states $\{0, a\}$, and transition probabilities $q, p$ for moves left and right respectively. Starting at vertex $z$, the probability of absorption at the origin 0 is

$$
\rho(z, a)=\frac{(q / p)^{z}-(q / p)^{a}}{1-(q / p)^{a}} \leq\left(\frac{q}{p}\right)^{z}
$$

provided $q \leq p$

Let $U_{\infty}=\left\{\exists t \geq 1: X_{t}=0\right\}$, i.e. the event that the particle ever returns to the root vertex in $\mathcal{T}$. It follows from (29) with $z=1$ and $a=\infty$ that

$$
\operatorname{Pr}\left(U_{\infty}\right)=\frac{1}{r-1}
$$

It follows that the expected number of visits by $\mathcal{X}$ to $v$ is $\frac{1}{1-\frac{1}{r-1}}=\rho$. We write

$$
R_{v}=\sum_{t=0}^{T} r_{t} \text { and } \rho=\sum_{t=0}^{\infty} \rho_{t}
$$


where $\rho_{t}=\operatorname{Pr}\left(X_{t}=v\right)$.

Now $r_{t}=\rho_{t}$ for $t \leq \ell_{1} / 2$ and part (a) follows once we prove that

$$
\sum_{t=\ell_{1} / 2+1}^{T} r_{t}=o(1) \text { and } \sum_{t=\ell_{1} / 2+1}^{\infty} \rho_{t}=o(1) .
$$

The first equation of (31) follows from

$$
\left|r_{t}-\frac{1}{n}\right| \leq \lambda_{\max }^{t}
$$

where $\lambda_{\max }$ is the second largest eigenvalue of the walk. This follows from (10).

The second equation of (31) is proved in Lemma 7 of [7] where it is shown that

$$
\sum_{t=\ell_{1} / 2+1}^{\infty} \rho_{t} \leq \sum_{2 j=\ell_{1} / 2+1}^{\infty}\left(\begin{array}{c}
2 j \\
j
\end{array}\right) \frac{(r-1)^{j}}{r^{2 j}} \leq \sum_{2 j=\ell_{1} / 2+1}^{\infty}\left(\frac{4(r-1)}{r^{2}}\right)^{j} .
$$

Thus

$$
R_{v}=\rho+O\left(T \lambda_{\max }^{\ell_{1} / 2}+T / n+(8 / 9)^{\ell_{1} / 2}\right)
$$

and part (a) follows. (b) We next note a property of random walks on undirected graphs which follows from results on electrical networks (see e.g. Doyle and Snell [11]). Let $v$ be a given vertex in a graph $G$ and $S$ a set of vertices disjoint from $v$. Let $p(G)$, the escape probability, be the probability that, starting at $v$, the walk reaches $S$ before returning to $v$. For an unbiased random walk,

$$
p=\frac{1}{d(v) R_{E F F}}
$$

where $R_{E F F}$ is the effective resistance between $v$ and $S$ in $G$. We assume each edge of $G$ has resistance 1 . In the notation of this paradigm, deleting an edge corresponds to increasing the resistance of that edge to infinity. Thus by Raleigh's Monotonicity Law, if edges are deleted from $G$ to form a sub-graph $G^{\prime}$ then $R_{E F F}^{\prime} \geq R_{E F F}$. So, if we do not delete any edges incident with $v$ then $p^{\prime} \leq p$.

It follows from (27) that $H$ becomes a tree after removing one edge. We can remove an edge not incident with $v$. By the above discussion on electrical resistance we see that this will not decrease $\operatorname{Pr}\left(U_{\infty}^{*}\right)$, where this is $U_{\infty}$ defined with respect to $\mathcal{T}^{*}$ which is $\mathcal{T}$ less one edge, not incident with $v$. We can argue crudely that

$$
\operatorname{Pr}\left(U_{\infty}^{*}\right) \leq \frac{1}{r}+\frac{r-1}{r} \cdot \frac{1}{r-1}=\frac{2}{r} .
$$

This is because there is an $\frac{r-1}{r}$ chance of a first move to a part of the tree that has branching factor $r-1$ at every vertex. 
Let $U_{1}^{*}=\left\{\mathcal{X}\right.$ returns to the root vertex after starting at $\left.\ell_{1} / 2\right\}$. Then, with $F_{T}$ equal to the probability of a return by $\mathcal{W}_{v}$ to $v$ during $[1, T]$, we have

$$
F_{T} \leq \operatorname{Pr}\left(U_{\infty}^{*}\right)+T \operatorname{Pr}\left(U_{1}^{*}\right)
$$

The RHS of (34) is at least the probability that $\mathcal{W}_{v}$ returns before reaching distance $\ell_{1} / 2$ or returns after reaching distance $\ell_{1} / 2$ at some time $t \leq T$.

Now, using (29), we see that

$$
\operatorname{Pr}\left(U_{1}^{*}\right) \leq \frac{1}{(r-1)^{\ell_{1} / 4}}
$$

Here we have $\ell_{1} / 4$ in place of $\ell_{1} / 2$ to account for the one place where we move left with probability $\frac{1}{r-2}$. We argue that at least one of the paths from $v$ to $w$ or $w$ to the boundary must be at least $\ell_{1} / 4$ and not use the vertex incident to the deleted edge.

Thus $F_{T} \leq(2+o(1)) / r$ and since $R_{v} \leq \frac{1}{1-F_{T}}$ we have $R_{v} \leq \frac{r+o(1)}{r-2}$.

\subsection{Proof of Theorem 4}

This section establishes the whp values of $|\mathcal{R}(t)|$ and $D_{s}(t)$ for $\Gamma(t)$. We also calculate the whp value of $|\mathcal{U}(t)|$, where $\mathcal{U}(t)$ is the number of unvisited edges at step $t$. This gives us the value of $\left|R_{t}\right|=2|\mathcal{U}(t)|$ (see Section 2), which we need for the proof of Theorem 5 .

Fix $v \in V$ and let $N(v)=\left\{w_{1}, w_{2}, \ldots, w_{r}\right\}$ and choose $0 \leq s \leq r$. Let

$$
P(v, s, t)=\operatorname{Pr}_{\mathcal{W}}\left(\left\{v, w_{1}, \ldots, w_{s}\right\} \subseteq \mathcal{R}(t) \text { and }\left\{w_{s+1}, \ldots, w_{r}\right\} \subseteq \mathcal{B}(t)\right) .
$$

Then

$$
\operatorname{Pr}_{\mathcal{W}}(v \in \mathcal{R}(t) \text { and has degree } s \text { in } \Gamma(t))=\left(\begin{array}{l}
r \\
s
\end{array}\right) P(v, s, t)
$$

Lemma 9 is only valid after the mixing time $T$, and Corollary 10 gives precise results only after some $t$ sufficiently larger than $T$. We assume henceforth that $t \geq \log ^{3} n$. We deal with the very beginning of the walk $(t<T)$ in Section 4.5. Define the set $\mathcal{R}_{T}(t)$ to be those vertices visited by the walk during steps $T, \ldots, t$, and let $\mathcal{B}_{T}(t)=V \backslash \mathcal{R}_{T}(t)$. Thus $\mathcal{R}(t)=\mathcal{R}_{T}(t) \backslash\left\{\mathcal{W}_{u}(0), \mathcal{W}_{u}(1), \ldots, \mathcal{W}_{u}(T-1)\right\}$

We recall the definition of a nice vertex, as given at the start of Section 4.1. We will say an edge $e=\{u, v\}$ is nice, if both $u, v$ are nice. The next lemma gives enough information to compute the expected number of unvisited nice vertices, edges between nice vertices, and the expected degree sequence of nice vertices in $\mathcal{R}(t)$. 
Lemma 13. Let $p_{t}=e^{-((r-2) t) /(\rho r n)}$ as given by $(3)$. $\mathbf{W h} \mathbf{p}_{G}{ }^{1}$, for all nice vertices $v$, and for all nice edges $\{u, v\}$, for $t \geq \log ^{3} n$,

(a) $\operatorname{Pr}_{\mathcal{W}}\left(v \in \mathcal{R}_{T}(t)\right)=(1+o(1)) e^{-\frac{t}{\rho n}}$.

(b) $\operatorname{Pr}_{\mathcal{W}}\left(\{u, v\} \in \mathcal{U}_{T}(t)\right)=(1+o(1)) e^{-\frac{2 t}{\rho r n}}$.

(c) Let

$$
P_{T}(v, s, t)=\operatorname{Pr}_{\mathcal{W}}\left(\left\{v, w_{1}, \ldots, w_{s}\right\} \subseteq \mathcal{R}_{T}(t) \text { and }\left\{w_{s+1}, \ldots, w_{r}\right\} \subseteq \mathcal{B}_{T}(t)\right)
$$

then

$$
P_{T}(v, s, t)=(1+o(1)) e^{-\frac{t}{\rho n}} p_{t}^{s}\left(1-p_{t}\right)^{r-s}
$$

Proof Part (a) follows directly from Corollary 10 using $p_{v}$ from (18) and the value of $R_{v}$ from $12(\mathrm{a})$.

Part (b) follows similarly, by subdividing the edge $e=\{u, v\}$ with an artificial vertex $\alpha$ to form the graph $H$. The stationary distribution of $\alpha$ in $H$ is $\pi_{\alpha}=2 /(r n+2)$, and the value of $R_{\alpha}$ is given by $R_{v}$ of Lemma $12(\mathrm{a})$.

For part (c) we proceed as follows. Let $X \subseteq N(v)$. Let $\gamma_{X}$ denote the contraction of $\{v\} \cup X$. Corollary 10 applies to $\gamma_{X}$ and

$$
p_{\gamma_{X}}=(1+o(1)) \frac{(r-2)(r+(r-2)|X|)}{r(r-1) n},
$$

as we now explain. From (18), the expression for $p_{\gamma_{X}} \sim \pi_{\gamma_{X}} / R_{\gamma_{X}}$. The stationary distribution of $\gamma_{X}$ is $\pi_{\gamma_{X}}=(|X|+1) / n$. Since $v$ is nice, the expected number of returns, is (up to a factor $1+o(1)), R_{\gamma_{X}}=\frac{1}{1-f}$; where $f$ is the probability of return to the root $\gamma_{X}$ of an infinite tree with branching factor $r-1$ at each non-root vertex. Thus $p_{\gamma_{X}}=(1+o(1)) \pi_{\gamma_{X}}(1-f)$. It remains to calculate $f$. At the root there are $|X|$ loops and $r-|X|+(r-1)|X|$ branching edges. This gives

$$
f=\frac{2|X|}{r(|X|+1)}+\frac{(r-2)|X|+r}{r(|X|+1)} \frac{1}{r-1},
$$

and hence (37) above.

Let $N(v)=\left\{w_{1}, w_{2}, \ldots, w_{r}\right\}$, and let $X=\left\{w_{1}, \ldots, w_{s}\right\} \cup Y$ where $Y \subseteq\left\{w_{s+1}, \ldots, w_{r}\right\}$. Then

$$
\begin{aligned}
\operatorname{Pr}_{\mathcal{W}}\left(\left\{v, w_{1}, \ldots, w_{s}\right\} \subseteq \mathcal{R}_{T}(t) \text { and }\left\{w_{s+1}, \ldots, w_{r}\right\} \subseteq \mathcal{B}_{T}(t)\right) \\
=\sum_{Y \subseteq\left\{w_{s+1}, \ldots, r\right\}}(-1)^{|Y|} \operatorname{Pr}_{\mathcal{W}}\left(\left(\left\{v, w_{1}, \ldots, w_{s}\right\} \cup Y\right) \subseteq \mathcal{R}_{T}(t)\right) \\
\quad=\sum_{Y \subseteq\left\{w_{s+1}, \ldots, r\right\}}(-1)^{|Y|} \frac{1+O\left(T \pi_{\gamma_{X}}\right)}{\left(1+p_{\gamma_{X}}\right)^{t}},
\end{aligned}
$$

\footnotetext{
${ }^{1}$ We use the subscript $G$ to emphasize that the probability space is random $r$-regular graphs
} 
where $|X|=0, \ldots, r-s$. If $s=0$, we suppose that $\left\{v, w_{1}, \ldots, w_{s}\right\}=\{v\}$.

Thus, for $s=0,1, \ldots, r$,

$$
\begin{aligned}
& \operatorname{Pr}_{\mathcal{W}}\left(\left\{v, w_{1}, \ldots, w_{s}\right\} \subseteq \mathcal{R}_{T}(t) \text { and }\left\{w_{s+1}, \ldots, w_{r}\right\} \subseteq \mathcal{B}_{T}(t)\right)= \\
& \quad \exp \left\{-(1+o(1)) \frac{(r-2)^{2} s+r(r-2)}{r(r-1) n} t\right\} \sum_{Y \subseteq[s+1, r]}(-1)^{|Y|} \exp \left\{-(1+o(1)) \frac{(r-2)^{2}|Y|}{r(r-1) n} t\right\} .
\end{aligned}
$$

There are two cases. When $t=O(n)$ we can write (39) as

$$
\exp \left\{-(1+o(1)) \frac{(r-2)^{2} s+r(r-2)}{r(r-1) n} t\right\}\left(1-\exp \left\{-\frac{(r-2)^{2}}{r(r-1) n} t\right\}\right)^{r-s}+o(1) \text {. }
$$

and (c) follows, since the terms above are $\Omega(1)$. When $t / n \rightarrow \infty$ we go back to (39) and observe that the sum is $1-o(1)$ and thus

$$
\begin{aligned}
\operatorname{Pr}_{\mathcal{W}}\left(\left\{v, w_{1}, \ldots, w_{s}\right\} \subseteq \mathcal{R}_{T}(t) \text { and }\left\{w_{s+1}, \ldots, w_{r}\right\} \subseteq \mathcal{B}_{T}(t)\right) & = \\
& (1+o(1)) \exp \left\{-\frac{(r-2)^{2} s+r(r-2)}{r(r-1) n} t\right\}
\end{aligned}
$$

as required.

\section{Proof of Theorem 4(a).}

Let $t \leq t_{0}-\omega n$, where $t_{0}=\rho n \log n$ and $\omega$ satisfies the conditions of Theorem 4 .

Using (26), (27) we have whp, that $|\overline{\mathcal{N}}|=O\left(n^{2 \epsilon_{1}} \log n\right)$, and thus $|\mathcal{N}|=n(1-o(1))$. Let $Z(t)=|\mathcal{R}(t) \cap \mathcal{N}|$. As there are at most $T=O(\log n)$ vertices in $\mathcal{R}(t) \backslash \mathcal{R}_{T}(t)$,

$$
\begin{aligned}
\mathbf{E} Z(t) & =n(1-o(1)) \mathbf{P r}_{\mathcal{W}}\left(v \in \mathcal{R}_{T}(t)\right)+O(T) \\
& =(1+o(1)) n e^{-\frac{t}{\rho n}}
\end{aligned}
$$

Thus $\mathbf{E} Z(t) \rightarrow \infty$ for $t \leq \rho n \log n-\omega n$.

We use the Chebyshev inequality to prove concentration of $Z(t)$ for a single $t \leq t_{0}-\omega n$.

Suppose that

$$
\log \log n \ll \omega^{\prime}=\omega^{\prime}(n) \ll \omega .
$$

We first show that

$$
\operatorname{Var}(Z(t))=O\left(r^{\omega^{\prime}} \mathbf{E} Z(t)\right)+e^{-a \omega^{\prime}} \mathbf{E}(Z(t))^{2},
$$

for some constant $a>0$. 
Fix $t$ and let $\mathcal{E}_{v}$ be the event that vertex $v \in \mathcal{R}(t)$. We claim that if $v, w$ are at distance at least $\omega^{\prime}$ then

$$
\operatorname{Pr}\left(\mathcal{E}_{v} \cap \mathcal{E}_{w}\right)=\left(1+e^{-\Omega\left(\omega^{\prime}\right)}\right) \operatorname{Pr}\left(\mathcal{E}_{v}\right) \operatorname{Pr}\left(\mathcal{E}_{w}\right) .
$$

To prove this we use Lemma 11. Let $S=\{v, w\}$, and let $\gamma(S)$ be the contraction of $S$. For the random walk on the associated $H$ we have

$$
\frac{1}{2}\left(R_{v}+R_{w}\right) \leq R_{\gamma} \leq \frac{1}{2}\left(R_{v}+R_{w}\right)\left(1+O\left(T e^{-\Omega\left(\omega^{\prime}\right)}\right)\right) .
$$

Indeed, the first move from $\gamma$ will be to a neighbour of $v$ or $w$. Assume it is to a neighbour of $v$. The expected number of returns will be $R_{v}$ plus $R_{w}$ times the probability of a visit to $w$ during the mixing time. Because $v$ and $w$ are at distance at least $\omega^{\prime}$, using (32), the probability of a visit to $w$ during $T$ can be bounded by $T\left(n^{-1}+\lambda_{\max }^{\omega^{\prime}}\right)$. Thus

$$
R_{\gamma}=\rho\left(1+e^{-\Omega\left(\omega^{\prime}\right)}\right), \quad \pi_{\gamma}=\frac{2}{n}, \quad p_{\gamma}=\left(1-O\left(T e^{-\Omega\left(\omega^{\prime}\right)}\right)\right) \frac{2}{\rho n} .
$$

Equation (44) follows on using Lemmas 9, 11 and equation (45).

Thus

$$
\begin{aligned}
\mathbf{E}\left(Z^{2}(t)\right) & =\mathbf{E} Z(t)+\sum_{\substack{v, w \\
\operatorname{dist}(v, w) \geq \omega^{\prime}}} \operatorname{Pr}\left(\mathcal{E}_{v} \cap \mathcal{E}_{w}\right)+\sum_{\substack{v, w \\
\operatorname{dist}(v, w)<\omega^{\prime}}} \operatorname{Pr}\left(\mathcal{E}_{v} \cap \mathcal{E}_{w}\right) \\
& \leq \mathbf{E} Z(t)+\left(1+e^{-a \omega^{\prime}}\right) \mathbf{E}(Z(t))^{2}+r^{\omega^{\prime}} \mathbf{E}(Z(t))
\end{aligned}
$$

and (43) follows. Applying the Chebyshev inequality we see that

$$
\operatorname{Pr}\left(|Z(t)-\mathbf{E} Z(t)| \geq \mathbf{E}(Z(t)) e^{-a \omega^{\prime} / 3}\right) \leq \frac{2 r^{\omega^{\prime}} e^{a \omega^{\prime}}}{\mathbf{E} Z(t)}+e^{-a \omega^{\prime} / 3}
$$

Provided $t \leq t_{0}-\omega n, \mathbf{E} Z(t) \geq e^{\omega / \rho} / 2$ and our choice of $\omega^{\prime}$ in (42) implies that the RHS of (46) is $o(1)$ for such $t$.

To prove the concentration of $Z(t)$ simultaneously for all $t \leq(1-\epsilon) t_{0}$, we proceed as follows. Let now $\omega^{\prime}=\ell_{1} / 2$ where $\ell_{1}=\epsilon_{1} \log _{r} n$ is given by (25). From (40)-(41), for $t \leq(1-\epsilon) \rho n \log n$, we have $\mathbf{E} Z(t)=(1+o(1)) n e^{-t / \rho n} \geq n^{\epsilon} / 2$. We see that (46) becomes

$$
\operatorname{Pr}\left(|Z(t)-\mathbf{E} Z(t)| \geq \mathbf{E}(Z(t)) e^{-a \omega^{\prime} / 3}\right) \leq \frac{2 r^{\omega^{\prime}} e^{a \omega^{\prime}} e^{t /(\rho n)}}{n}+e^{-a \omega^{\prime} / 3}=o\left(n^{-\delta}\right)
$$

for some (small) $\delta>0$ constant. We can make $\delta=\left(a \epsilon_{1} \log r\right) / 4$ provided we make $\epsilon>$ $\epsilon_{1}\left(1+\frac{a}{\log r}\right)$.

We interpolate $\left[0, t_{0}\right]$ at $M=n^{\delta / 2}$ integer points $s_{1}, \ldots, s_{M}$ a distance $\sigma=t_{0} n^{-\delta / 2}$ apart (ignoring rounding). Let $\mathcal{H}(M)$ be the event that $\left\{|Z(t)-\mathbf{E} Z(t)| \leq \mathbf{E}(Z(t)) e^{-a \omega^{\prime} / 3}\right\}$ holds simultaneously at $s_{1}, \ldots, s_{M}$. Then $\operatorname{Pr}(\neg \mathcal{H}(M))=o\left(n^{-\delta / 2}\right)=o(1)$. 
The value of $Z(t)$ is non-increasing, with $\mathbf{E} Z(t)=(1+o(1)) n e^{-t / \rho n}$. Thus for any $t, s_{i} \leq t \leq$ $s_{i+1}$,

$$
\begin{aligned}
Z\left(s_{i}\right) & \geq Z(t) \geq Z\left(s_{i+1}\right) \\
(1+o(1)) e^{\left(t-s_{i}\right) / \rho n} & \geq \frac{Z(t)}{\mathbf{E} Z(t)} \geq(1-o(1)) e^{\left(t-s_{i+1}\right) / \rho n} .
\end{aligned}
$$

But $e^{\left(t-s_{i}\right) / \rho n}, e^{\left(t-s_{i+1}\right) / \rho n}$ are both $1-o(1)$ and so the concentration result holds for nice vertices for $t \leq(1-\epsilon) t_{0}$.

Lemma 13 applies only to nice vertices. We next consider $\overline{\mathcal{N}} \cap \mathcal{R}(t)$. It follows from Lemmas 9 and 12 that

$$
\text { whp } \overline{\mathcal{N}} \subseteq \mathcal{B}_{T}(t) \text { for } t \geq 10 \epsilon_{1} n \log n .
$$

However for $t \leq 10 \epsilon_{1} n \log n$,

$$
\mathbf{E} Z(t) \geq n^{1-10 \epsilon_{1}} \gg|\overline{\mathcal{N}}| \geq|\overline{\mathcal{N}} \cap \mathcal{R}(t)|
$$

This completes the proof of part (a) of Theorem 4.

\section{Proof of Theorem 4(b).}

The proof of part (b) is similar to that of (a). Observe first that part (a) and Lemma 13 imply

$$
\mathbf{E}\left(D_{s}^{\prime}(t)\right)=(1+o(1)) N_{t}\left(\begin{array}{l}
r \\
s
\end{array}\right) p_{t}^{s}\left(1-p_{t}\right)^{r-s}
$$

where $D_{s}^{\prime}(t)$ is the number of nice vertices of degree $s$ in $\Gamma(t)$.

Our next aim is to show

$$
\mathbf{E}\left(D_{s}^{\prime}(t)\right)=e^{\Omega(\omega)} \text { for }\left(\tau_{r-s}\right)^{1+\delta} \leq t \leq t_{s}-\omega n
$$

As a function of $t, N_{t} p_{t}^{s}\left(1-p_{t}\right)^{r-s}$ is log-concave and to bound $\mathbf{E}\left(D_{s}^{\prime}(t)\right)$ from below we only need to check $\mathbf{E}\left(D_{s}^{\prime}(t)\right)$ at the lower bound $t=\left(\tau_{r-s}\right)^{1+\delta}$ and at the upper bound $t=t_{s}-\omega n$. See (6) for the definitions of $\tau_{s}, t_{s}$. For $t=o(n)$ we have $p_{t}=1-o(1)$ and $N_{t} \sim n$ and then (51) is simple to verify at the lower bounds for $t$ in (51). When $t=t_{s}-\omega n$ we have $p_{t}=e^{\frac{\omega(r-2)}{\rho r}} n^{-\frac{r-2}{s(r-2)+r}}$ and $N_{t}=e^{\frac{\omega}{r}} n^{1-\frac{r}{s(r-2)+r}}$ and (51) is also easy to verify.

We can use the Chebyshev inequality to prove concentration. We let $\mathcal{F}_{v}(s)$ be the event that $v$ is a vertex of degree $s$ in $\Gamma(t)$. We prove that for $v, w \in \mathcal{N}$,

$$
\operatorname{Pr}\left(\mathcal{F}_{v}(s) \cap \mathcal{F}_{w}(s)\right)=\left(1+e^{-\Omega\left(\omega^{\prime}\right)}\right) \operatorname{Pr}\left(\mathcal{F}_{v}(s)\right) \operatorname{Pr}\left(\mathcal{F}_{w}(s)\right)
$$

whenever $v, w$ are at least $\omega^{\prime}$ apart, where $\omega^{\prime} / \log \log n \rightarrow \infty$ and $\omega=o(\log n)$ and $\omega^{\prime}=o(\omega)$.. We can argue for this by a small change in the argument for (44). This proves concentration 
for $\left(\tau_{r-s}\right)^{1+\delta} \leq t \leq t_{s+1}-\omega n$. Let $D_{\geq s}(t)=\sum_{k=s}^{r} D_{k}(t)$. This sum is monotone non-increasing with $t$. Simultaneous concentration of $D_{\geq s}(t)$ and hence $D_{s}(t)$ follows from the interpolation method of part (a) applied to $D_{\geq s}(t)$.

We must now argue that the contribution of $\overline{\mathcal{N}}$ is negligible. Equation (48) shows that whp $\overline{\mathcal{N}} \subseteq \mathcal{B}(t)$ for $t \geq T_{1}=10 \epsilon_{1} n \log n$. On the other hand, by $(50)$, for $t \leq T_{1}$ and any $0 \leq s \leq r$,

$$
\mathbf{E}\left(D_{s}^{\prime}\left(T_{1}\right)\right)=\Omega\left(n^{1-\frac{10 \epsilon_{1}(r+s(r-2))}{\rho r}}\right) \gg n^{2 \epsilon_{1}} .
$$

It follows from this and concentration of $D_{s}^{\prime}(t)$ and $(26)$ that $D_{s}^{\prime}(t) \sim D_{s}(t)$ whp and the proof of part (b) is complete.

\subsection{Proof of Theorem 3}

We combine Lemma 7 and Theorem 4 with the results of Molloy and Reed [19, 20]. We summarize what we need from these two papers:

Theorem 14. Let $\lambda_{0}, \lambda_{1}, \ldots, \lambda_{r} \in[0,1]$ be such that $\lambda_{0}+\lambda_{1}+\cdots+\lambda_{r}=1$. Suppose that $\mathbf{d}=\left(d_{1}, d_{2}, \ldots, d_{N}\right)$ satisfies $\left|\left\{j: d_{j}=s\right\}\right|=(1+o(1)) \lambda_{s} N$ for $s=0,1, \ldots, r$. Let $G_{n, \mathbf{d}}$ be chosen randomly from graphs with vertex set $[N]$ and degree sequence $\mathbf{d}$. Let

$$
L=\sum_{s=1}^{r} s(s-2) \lambda_{s}
$$

(a) If $L<0$ then whp $G_{n, \mathbf{d}}$ is sub-critical.

(b) If $L>0$ then whp $G_{n, \mathbf{d}}$ is super-critical. Furthermore the unique giant component has size $\theta n$ where $\theta$ is defined as follows: Let $\Lambda=\sum_{s=1}^{r} s \lambda_{s}$. Define $\alpha$ to be the smallest positive solution to

$$
\Lambda-2 \alpha-\sum_{s=1}^{r} s \lambda_{s}\left(1-\frac{2 \alpha}{\Lambda}\right)^{s / 2}=0
$$

Then

$$
\theta=1-\sum_{s=0}^{r} \lambda_{s}\left(1-\frac{2 \alpha}{\Lambda}\right)^{s / 2}
$$

We now evaluate $L$ in the context of $\Gamma(t)$. Then Theorem 4 implies that we can take $\lambda_{s}=$ $\left(\begin{array}{l}r \\ s\end{array}\right) p_{t}^{s}\left(1-p_{t}\right)^{r-s}$ so that

$$
L=\sum_{s=0}^{r}\left(\begin{array}{l}
r \\
s
\end{array}\right) p_{t}^{s}\left(1-p_{t}\right)^{r-s} s(s-2)=r p_{t}\left((r-1) p_{t}-1\right)
$$


Thus the critical value for $t$ is the one that gives $L=0$ and $p_{t}=\frac{1}{r-1}$. One can easily check that this is indeed the case for $t^{*}$ as defined in (1). Intuitively, this can be seen as follows. When we grow components outward from a given vertex, the branching factor is $(r-1) p_{t}$, so when $(r-1) p_{t}<1$, all components are of finite size.

Parts (i) and (ii) of Theorem 3 follow. We next check the claimed size of $C_{1}(t)$. First of all, $\Lambda=r p_{t}>0$. We divide (53) by $\Lambda$ and then let $\phi=1-\frac{2 \alpha}{\Lambda}$ so that (53) becomes (5), and (54) becomes (4). The equation $\phi=\left(1-p+p \phi^{1 / 2}\right)^{2(r-1)}$ for $\phi$, has one solution at $\phi=1$ and a possible further solution $0<\phi<1$, which determines the size of the giant component. With this value for $\phi$ we see that and then whp $\left|C_{1}(t)\right| \sim \theta N_{t}$ as claimed.

To prove (iii) we use the result of Hatami and Molloy [15] that when $|L|=O\left(n^{-1 / 3}\right)$ the size of the giant is $n^{2 / 3+o(1)}$. At each step, at most $r$ vertices in $\Gamma(t)$ change their degree and so $L$ changes by $O(1 / n)$. $L$ starts out at $r(r-2)$ and so at some time it becomes equal to $O\left(n^{-1 / 3}\right)$. This will happen at $t \sim t^{*}$ and the conditions of [15] will be satisfied. At this point whp there are $\Theta(n)$ vertices in $\Gamma(t)$ and (iii) follows.

\subsection{Proof of Theorem 5}

In this section we study the number of components of a given size. In principle one should be able to work this out from Lemma 7 and Theorem 4. This has proven more difficult than we anticipated. Instead, we try to estimate the number directly. We can use these lemmas though to argue that almost all small components are trees. Indeed if we fix $t$ and condition on the values $D_{s}=D_{s}(t)$ satisfying Theorem 4 then we have the following:

\section{Lemma 15.}

(a) If $t \leq \epsilon_{1} n \log n$ then whp there are at most $n^{3 \epsilon_{1}}$ components of size $k \leq \epsilon_{1} \log n$ that are not trees.

(b) If $t \geq \epsilon_{1} n \log n$ then whp there are no components of size $k \leq \log ^{2} n$ that are not trees.

Proof Let $N=|\mathcal{R}(t)|$. Applying Lemma 7 we see that the expected number of sets of $k$ vertices that contain at least $k$ edges is bounded by

$$
\left(\begin{array}{c}
N \\
k
\end{array}\right)\left(\begin{array}{c}
k \\
2 \\
k
\end{array}\right)\left(\frac{r}{n}\right)^{k} \leq\left(\frac{r N e^{2}}{2 n}\right)^{k} .
$$

To prove (a) we take $N=n$ and apply the Markov inequality. To prove (b) we take $N=N_{t}$.

With this in mind we concentrate on the number of tree components of size $k$ for some $k \leq \epsilon_{1} \log n$. Since there are whp at most $n^{2 \epsilon_{1}}$ vertices that are not nice, we will concentrate 
on counting the number of components that are made up of nice vertices only. We will also assume that $t \leq t_{0}$, see $(51)$.

The following is Lemma 4 of [2].

Lemma 16. [2] Let $\mathcal{T}$ be a labeled infinite $r$-regular tree. Let $b_{k}$ be the number of labeled subtrees of size $k$ rooted at vertex $v$ of $\mathcal{T}$. Then

$$
b_{k}=\frac{r}{(r-2) k+2}\left(\begin{array}{c}
(r-1) k \\
k-1
\end{array}\right) \text {. }
$$

Lemma 16 counts labeled sub-trees. Let $T$ be such a tree. Each edge $e=(x, y)$, with $x$ closest to $v$, is associated with a label $\lambda_{e}$ indicating that it is the $\lambda_{e}$-th edge incident with $x$, in numerical order. Now consider the situation described in Lemma 8. Fix $v \in \mathcal{R}(t) \cap \mathcal{N}$. Assuming $v \in \mathcal{N}$ introduces some conditioning on the allowable pairings described in Lemma 8. However $\operatorname{Pr}(v \in \mathcal{N})=1-O\left(n^{2 \epsilon_{1}-1}\right)$ (see (26)) and then we can use

$$
\frac{\operatorname{Pr}(A)-\operatorname{Pr}(\neg B)}{\operatorname{Pr}(B)} \leq \operatorname{Pr}(A \mid B) \leq \frac{\operatorname{Pr}(A)}{\operatorname{Pr}(B)}
$$

for events $A, B$ to correct (55) below for this conditioning. Consider the $k$ neighbourhood of $v$ in the multi-graph on $[n]$ induced by a random pairing on $R(t)$. It is a tree. Now delete any edge that corresponds to an edge $(x, y)$ with $x \in \mathcal{R}(t), y \in \mathcal{B}(t)$. Let $T$ be the component that contains $v$. If $T$ has $k$ vertices then $T$ corresponds to a tree component of $\Gamma(t)$ with $k$ vertices.

So, fix a tree $T^{*}$ as counted in Lemma 16 and let us determine the probability that $T=T^{*}$. The probability space for this calculation is as follows: Let $F_{t}, R_{t}$ be as in Lemma 8 . We have paired up the elements of $F_{t}$ and we are now considering random pairings of $R_{t}$. To extend $F_{t}$ to $F$, we can generate the pairing of $R_{t}$ in any order we please. Thus we start by pairing elements of $W_{v}$ the root of our tree.

If we choose $x \in W_{v}$ then the probability it is paired with $y \in W_{z}$, for some $z \in \mathcal{R}(t)$, is $\nu=\frac{r|\mathcal{R}(t)|-1}{\left|R_{t}\right|-1}$. Using Lemma (13)(a),(b), with $|\mathcal{R}(t)|=(1+o(1)) N_{t}$ and $\left|R_{t}\right|=2|\mathcal{U}(t)|$ we obtain

$$
\nu=\frac{r|\mathcal{R}(t)|-1}{\left|R_{t}\right|-1}=(1+o(1)) e^{-(r-2) t /(\rho r n)}=(1+o(1)) p_{t},
$$

where $p_{t}$ is given by (3), and the $o(1)$ term is $o\left(\log ^{-K} n\right)$ for any positive constant $K$.

Suppose now that we have generated $O(\log n)$ pairings. Then both $|\mathcal{R}(t)|$ and $\left|R_{t}\right|$ change by $O(\log n)$ and, provided $t \leq t_{0}(1-\epsilon), \epsilon>0$, since they are both of size $\Omega(n)$. Choosing 
an unpaired $x^{\prime} \in W_{v^{\prime}}$ for $v^{\prime} \in \mathcal{R}(t)$ we see that the probability of $x$ being paired with some $y^{\prime} \in W_{z^{\prime}}$ where $z^{\prime} \in \mathcal{R}(t)$, is again $(1+o(1)) p_{t}$.

To estimate $\operatorname{Pr}\left(T=T^{*}\right)$ we start at the root $v$ and examine the points paired with $W_{v}$, where $W_{v} \subseteq R_{t}$, because $v \in \mathcal{R}(t)$. The count in Lemma 16 assumes an ordering of the neighbours of each vertex and by implication an ordering of $W_{v}$ and a statement about which members of $W_{v}$ are paired with $W_{\mathcal{R}(t)}$ and which should not. Suppose we pair $W_{v}$ with points from $W_{x_{i}}, i=1,2, \ldots, d$. Then we continue by pairing up $W_{x_{1}}$ and then $W_{x_{2}}$ and so on. The factor $p_{t}^{k-1}$ is from the $k-1$ times we have to pair with $W_{\mathcal{R}(t)}$ and the factor $\left(1-p_{t}\right)^{(r-2) k+2}$ is from the number of times we do not.

It follows that

$$
\operatorname{Pr}\left(T=T^{*}\right)=(1+o(1)) p_{t}^{k-1}\left(1-p_{t}\right)^{(r-2) k+2}
$$

It follows from (56) that

$$
\mathbf{E}(N(k, t))=(1+o(1)) N_{t} \frac{b_{k}}{k} p_{t}^{k-1}\left(1-p_{t}\right)^{(r-2) k+2} .
$$

It remains to prove concentration around the mean. We use the Chebyshev inequality. We fix two vertex disjoint trees $T_{1}, T_{2}$ in $G$. Arguing as above we see that

$$
\operatorname{Pr}\left(T_{1}, T_{2} \text { are components of } \Gamma(t)\right) \leq(1+o(1)) \prod_{i=1}^{2} \operatorname{Pr}\left(T_{i} \text { is a component of } \Gamma(t)\right) \text {. }
$$

Provided $\mathbf{E}(N(k, t)) \rightarrow \infty$ and it does so for $\left(\tau_{k(r-2)+2}\right)^{1+\delta} \leq t \leq(1-\epsilon) t_{k}$ we can use the Chebychev inequality to prove Theorem 5(a) for any fixed $t$. Note that here we have used concentration in the configuration model to imply concentration in the simple graph model.

We now prove Theorem $5(\mathrm{~b})$. For $k$ constant, $\mathbf{E}(N(k, t))=(1+o(1)) \eta(k, t) \geq n^{\epsilon^{\prime}}$, throughout the range $\epsilon n \leq t \leq(1-\epsilon) t_{k-1}$. Concentration can be established by the methods used in Section 4.2. Let $A$ be a large constant. For $\epsilon n \leq t \leq A n$ we use the Chebychev inequality directly, and for $A n \leq t \leq(1-\epsilon) t_{k-1}$ we use the interpolation method. For $\epsilon n \leq t \leq A n$, $\mathbf{E} N(k, t) \geq a_{k} n$ where $a_{k}$ is some constant. Using the Chebychev inequality as in (46), but with the factor $e^{-a \omega^{\prime} / 3}$ replaced by $o\left(n^{-\delta}\right)$, for some $\delta>0$, (which we can do because of the size of $\mathbf{E} N(k, t)$ in this range),

$$
\operatorname{Pr}\left(|N(k, t)-\mathbf{E} N(k, t)| \geq \mathbf{E} N(k, t) o\left(n^{-\delta}\right)\right)=o\left(n^{-\delta}\right) .
$$

Interpolate $\epsilon n \leq t \leq A n$ at $h=n^{\delta / 2}$ points $\left(t_{1}, \ldots, t_{h}\right), \ell=(A-\epsilon) n^{1-\delta / 2}$ apart. Let $t \in$ $\left(t_{j}, t_{j+1}\right)$. Then $\left|N(k, t)-N\left(k, t_{j}\right)\right| \leq r \ell$ and $\left|\eta(k, t)-\eta\left(k, t_{j}\right)\right|=O(\ell)$. It follows that, with probability $1-o\left(n^{-\delta / 2}\right),|N(k, t)-\mathbf{E} N(k, t)| \leq \mathbf{E} N(k, t) o\left(n^{-\delta / 2}\right)$ for all $\epsilon n \leq t \leq A n$. For $A N \leq t \leq(1-\epsilon) t_{k-1}$ we use the interpolation method of Section 4.2 as follows. Whp the maximum component size is $O(\log n)$ and there are $O(\log n)$ vertices on non-tree components. 
Let $M(k, t)$ be the number of vertices on components of size at least $k$. Then $M(k, t)$ is monotone non-increasing and

$$
\begin{aligned}
M(k, t) & =\sum_{j=k}^{O(\log n)} j N(j, t)+O(\log n) \\
k N(k, t) & =M(k-1, t)-M(k, t)+O(\log n) .
\end{aligned}
$$

Applying the interpolation method to $M(k, t)$ will complete the proof of Theorem 5(b).

\subsection{In the beginning}

We remind the reader that our proofs so far, we have assumed $t \geq \log ^{3} n$. We now consider the first few moves of the walk. Using Lemma 7 we see that for $1 \leq t \leq \log ^{3} n$ we have that $\Gamma(t)$ is a random graph with a degree sequence of the following form: There are $n-s$ vertices of degree $r$, where $s \leq r t$, and $s$ vertices of degree $<r$. If the minimum degree in $\Gamma(t)$ is at least one then whp we find that $\Gamma(t)$ is connected. Indeed, let $V_{r}$ be the set of vertices of degree $r$ in $\Gamma(t)$. We argue that whp

$$
\begin{aligned}
& V_{r} \text { induces a connected subgraph of } \Gamma(t) \text {. } \\
& \text { Each } x \in \mathcal{R}(t) \backslash V_{r} \text { is adjacent to } V_{r} .
\end{aligned}
$$

For $k$ even let

$$
\phi(k)=\frac{k !}{(k / 2) ! 2^{k / 2}}
$$

be the number of ways of partitioning $[k]$ into $k / 2$ pairs.

Let $m=O\left(\log ^{3} n\right)$ be the sum of the degrees, in $\Gamma(t)$, of the vertices in $\mathcal{R}(t) \backslash V_{r}$. Then

$$
\begin{aligned}
\operatorname{Pr}((57) \text { fails }) & \leq \sum_{k=3}^{n / 2} \sum_{l=0}^{m}\left(\begin{array}{l}
n \\
k
\end{array}\right)\left(\begin{array}{c}
m \\
l
\end{array}\right) \frac{\phi(k r+l) \phi((n-s-k) r+m-l)}{\phi((n-s) r+m)} \\
& =\sum_{k=3}^{n / 2} \sum_{l=0}^{m}\left(\begin{array}{l}
n \\
k
\end{array}\right)\left(\begin{array}{c}
m \\
l
\end{array}\right) \frac{\left(\begin{array}{c}
(r(n-s)+m) / 2 \\
(k r+l) / 2
\end{array}\right)}{\left(\begin{array}{c}
r(n-s)+m \\
k r+l
\end{array}\right)} \\
& \leq \sum_{k=3}^{n / 2} \sum_{l=0}^{m}\left(\begin{array}{l}
n \\
k
\end{array}\right)\left(\begin{array}{c}
m \\
l
\end{array}\right) \frac{1}{\left(\begin{array}{c}
(r(n-s)+m) / 2 \\
(k r+l) / 2
\end{array}\right)} \\
& \leq \sum_{k=3}^{n / 2} \sum_{l=0}^{m}\left(\begin{array}{l}
n \\
k
\end{array}\right)\left(\begin{array}{c}
m \\
l
\end{array}\right)\left(\frac{k r+l}{r(n-s)+m}\right)^{(k r+l) / 2} \\
& =o(1) .
\end{aligned}
$$


Explanation of (59): Choose a set of $k$ vertices $S$ of degree $r$ and $l$ points from the $m$ points in $W$ associated with vertices $T$ of degree less than $r$. (Some of the points associated with these latter vertices have already been paired). Now pair up the $k r+l$ points randomly and the remaining points randomly. The $l$ points contain the edges between $S$ and $T$.

The probability that (58) fails is $O(\log n / n)$. A vertex of $\mathcal{R}(t) \backslash V_{r}$ of degree $d$ has an $O\left(n^{-d}\right)$ chance of not being connected to $V_{r}$.

So we only have to deal with the possibility that there are isolated vertices in $\Gamma(t)$ for $t \leq \log ^{3} n$. So consider the event

$$
\mathcal{A}(t)=\left\{\exists v \in N\left(W_{t}\right): v \in \mathcal{R}(t) \text { and } N(v) \subseteq \mathcal{B}(t)\right\} .
$$

We claim that

$$
\operatorname{Pr}(\mathcal{A}(t))=O\left(\frac{\log ^{3} n}{(r-1)^{\ell_{1} / 2}}\right)
$$

It follows that

$$
\operatorname{Pr}((58) \text { fails }) \leq \sum_{t=1}^{\log ^{3} n} \operatorname{Pr}(\mathcal{A}(t))=o(1) .
$$

To prove (60) fix $t$ and a neighbour $v$ of $W_{t}$. Equation (27) implies that there is at least one neighbour $w$ of $v$ that is not contained in a small cycle. If $w \neq X_{t}$ then to reach $w$ the walk $\mathcal{W}$ must emulate a walk on the infinite tree $\mathcal{T}$ that starts at distance $\ell_{1} / 2$ from the root and visits it. This has probability $1 /(r-1)^{\ell_{1} / 2}$ and this must be inflated by $\log ^{3} n$ to account for $\log ^{3} n$ possible starting times. If $w=X_{t}$ then to visit another neighbour of $v$ then we must first reach distance at least $\ell_{1} / 2$ and then we can repeat the argument and use inequality (35).

\section{The evolution of $\Gamma(t)$ in $G_{n, p}$ and $D_{n, p}$}

We first consider $G_{n, p}$ and prove Theorem 1.

We first note some properties of the degree sequence $d_{G}(v)$ of $G_{n, p}$. We assume that $c=n^{1 / \omega}$ where $\omega \rightarrow \infty$. Let $\omega_{1}=\log ^{1 / 3} n$. For a fixed vertex $v$, its degree $d_{G}(v)$ satisfies

$$
\operatorname{Pr}\left(\left|d_{G}(v)-c \log n\right| \geq \omega_{1}(c \log n)^{1 / 2}\right) \leq 2 e^{-\omega_{1}^{2} / 3} .
$$

This follows from Chernoff bounds on the tail of the binomial distribution. Denote by $\mathcal{N}_{d}$, vertices of $G_{n, p}$ which have degrees in the range $c \log n \pm \omega_{1}(c \log n)^{1 / 2}$. By the Markov inequality, we see that whp $\left|\neg\left(\mathcal{N}_{d}\right)\right|=\left(n e^{-\omega_{1}^{2} / 4}\right)$.

Let $|\mathcal{R}(t)|=N$. Because $\Gamma(t)$ has the distribution $G_{N, p}$, we only need good estimates of $N$. We can get these using Lemma 9. Fix a vertex $v \in \mathcal{N}_{d}$. It is shown in [9] that whp 
$R_{v}=1+O(1 / \log n)$ for all $v \in V$. Let $\left.\epsilon=1 /(\log n)^{1 / 6}\right)$, then for $v \in \mathcal{N}_{d}, \pi_{v}=(1+O(\epsilon))(1 / n)$. Thus $p_{v}=(1+O(\epsilon))(1 / n)$.

For $t \geq \log ^{3} n,(22)$ implies that

$$
\operatorname{Pr}(v \in \mathcal{R}(t))=(1+O(\log n / n)) e^{-(1+O(\epsilon)) t / n} .
$$

Recall that $t_{\theta}=n(\log \log n+(1+\theta) \log c)$, and assume that $t=t_{\theta}$, where $\theta=O(1)$ then

$$
\mathbf{E}\left(\left|\mathcal{N}_{d} \cap \mathcal{R}(t)\right|\right)=(1+o(1)) \frac{n}{c^{1+\theta} \log n} .
$$

Regarding concentration, we can argue as in the proof (44) that if $v, w \in \mathcal{N}_{d}$ are at distance at least $\omega_{1} / 2$ in $G$ then

$$
\operatorname{Pr}\left(\mathcal{E}_{v} \cap \mathcal{E}_{w}\right)=(1+o(1)) \operatorname{Pr}\left(\mathcal{E}_{v}\right) \operatorname{Pr}\left(\mathcal{E}_{w}\right)
$$

where $\mathcal{E}_{v}=\{v \in \mathcal{R}(t)\}$.

Then if $X=\left|\mathcal{R}(t) \cap \mathcal{N}_{d}\right|$ then

$$
\begin{aligned}
& \mathbf{E}(X(X-1)) \leq \sum_{v \in \mathcal{N}_{d}} \sum_{\substack{w \in \mathcal{N}_{d} \\
\operatorname{dist}(v, w) \geq \omega_{1} / 2}} \operatorname{Pr}\left(\mathcal{E}_{v} \cap \mathcal{E}_{w}\right)+\sum_{v \in \mathcal{N}_{d}} \sum_{\substack{w \in \mathcal{N}_{d} \\
\text { dist }(v, w) \leq \omega_{1} / 2}} \operatorname{Pr}\left(\mathcal{E}_{v} \cap \mathcal{E}_{w}\right) \\
& \leq(1+o(1)) \sum_{v \in \mathcal{N}_{d}} \sum_{\substack{w \in \mathcal{N}_{d} \\
\operatorname{dist}(v, w) \geq \omega_{1} / 2}} \operatorname{Pr}\left(\mathcal{E}_{v}\right) \operatorname{Pr}\left(\mathcal{E}_{w}\right)+\sum_{v \in \mathcal{N}_{d}} \sum_{\substack{w \in \mathcal{N}_{d} \\
\text { dist }(v, w) \leq \omega_{1} / 2}} \operatorname{Pr}\left(\mathcal{E}_{v}\right) \\
& \leq(1+o(1)) \mathbf{E}(X)^{2}+O\left((c \log n)^{\omega_{1} / 2}\right) \mathbf{E}(X)
\end{aligned}
$$

which implies that $\operatorname{Var}(X)=o\left(\mathbf{E}(X)^{2}\right)$ and then the Chebyshev inequality implies that $X \sim \mathbf{E}(X) \sim \frac{n}{c^{1+\theta} \log n}$ whp.

The vertices outside $\mathcal{N}_{d}$ only contribute $o(N)$ whp and thus

$$
N\left(t_{\theta}\right)=(1+o(1)) \mathbf{E} N\left(t_{\theta}\right)=(1+o(1)) n /\left(c^{1+\theta} \log n\right) .
$$

The threshold for the giant component in $G_{N, p}$ is at $N p=1$, i.e. as $\theta \rightarrow 0$ from below. Theorem 1 follows immediately from this.

We next consider $D_{n, p}$ and prove Theorem 2. As the details are similar to those above, our discussion will be brief. If $n p=c \log n$ and $(c-1) \log n \rightarrow \infty$ then whp $D_{n, p}$ is strongly connected, so a random walk on $D_{n, p}$ is ergodic. It was established in [10] that if $(c-1) \log n \rightarrow$ $\infty$, then almost all vertices $v$ have have stationary distribution $\pi_{v}=(1+o(1)) / n$. By the method of deferred decisions, $\vec{\Gamma}(t)$ is a random digraph $D_{N(t), p}$ on $N(t)=|\mathcal{R}(t)|$ vertices, where $N(t)$ is given as above. It was proved in [17] that the threshold for the emergence of a giant strongly connected component in $D_{n, p}$ is at $n p \sim 1$. Theorem 2 follows. 


\section{References}

[1] I. Benjamini and A. Sznitman, Giant Component and Vacant Set for Random Walk on a Discrete Torus. J. Eur. Math. Soc., 10, 1, 1-40, (2008).

[2] A. Beveridge, A.M. Frieze and C.J.H. McDiarmid, Random minimum length spanning trees in regular graphs, Combinatorica 18 (1998) 311-333.

[3] B.Bollobás, A probabilistic proof of an asymptotic formula for the number of labelled regular graphs, European Journal on Combinatorics 1 (1980) 311-316.

[4] E.A.Bender and E.R.Canfield, The asymptotic number of labelled graphs with given degree sequences, Journal of Combinatorial Theory, Series A 24 (1978) 296-307.

[5] J. Černy, A. Teixeira and D. Windisch, Giant vacant component left by a random walk in a random $d$-regular graph. Annales de l'Institut Henri Poincaré (B) Probabilités et Statistiques (to appear).

[6] J. Črny and A. Teixeira, Critical window for the vacant set left by random walk on random regular graphs, arXiv:1101.1978.

[7] C. Cooper and A. M. Frieze, The cover time of random regular graphs, SIAM Journal on Discrete Mathematics, 18 (2005) 728-740.

[8] C. Cooper and A. M. Frieze, The cover time of the preferential attachment graph. Journal of Combinatorial Theory Series B, 97(2) 269-290 (2007).

[9] C. Cooper and A. M. Frieze, The cover time of the giant component of of $G_{n, p}$. Random Structures and Algorithms, 32, 401-439 J. Wiley (2008).

[10] C. Cooper and A. M. Frieze, Stationary distribution and cover time of random walks on random digraphs, arXiv:1103.4317.

[11] P. G. Doyle and J. L. Snell Random Walks and Electrical Networks. Carus Mathematical Monograph 22, AMA (1984).

[12] W. Feller, An Introduction to Probability Theory, Volume I, (Second edition) Wiley (1960).

[13] J. Friedman, A proof of Alon's second eignevalue conjecture and related problems, Memoirs of the American Mathematical Society 195, 2008.

[14] A. Frieze and B. Pittel, Perfect matchings in random graphs with prescribed minimal degree, Trends in Mathematics, Birkhauser Verlag, Basel (2004), 95-132.

[15] H. Hatami and M. Molloy, The scaling window for a random graph with a given degree sequence, Proceedings of SODA 2010. 
[16] M. Jerrum and A. Sinclair. The Markov chain Monte Carlo method: an approach to approximate counting and integration. In Approximation Algorithms for NTP-hard Problems. (D. Hochbaum ed.) PWS (1996) 482-520

[17] R. Karp, The transitive closure of a random digraph, Random Structures and Algorithms, 1, 73-93 J. Wiley (1990).

[18] C. McDiarmid, Concentration, in: Probabilistic Methods for Algorithmic Discrete Mathematics, Algorithms Combin. 16, Springer, Berlin (1998), 195-248.

[19] M. Molloy and B. Reed, A Critical Point for Random Graphs with a Given Degree Sequence Random Structures and Algorithms 6 (1995) 161-180.

[20] M. Molloy and B. Reed, The Size of the Largest Component of a Random Graph on a Fixed Degree Sequence, Combinatorics, Probability and Computing 7 (1998) 295-306.

[21] D. Windisch, Logarithmic components of the vacant set for random walk on a discrete torus, Electronic Journal of Probability. 13 (2008), Paper 28, pages 880-897. 